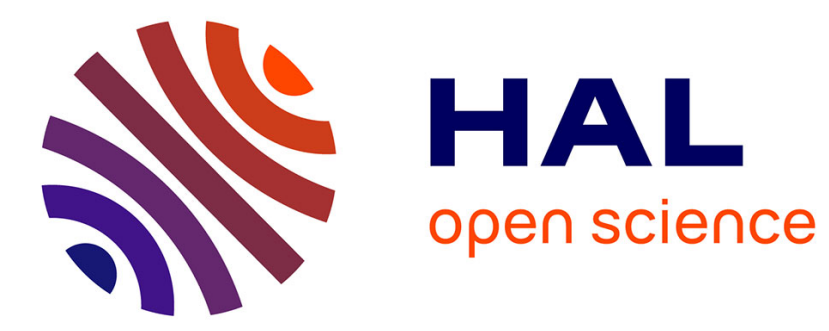

\title{
Building a global time on parallel machines
}

\author{
Jean-Marc Jézéquel
}

\section{To cite this version:}

Jean-Marc Jézéquel. Building a global time on parallel machines. Proc. of the 3rd International Workshop on Distributed Algorithms, Sep 1989, Nice, France. hal-00765061

\section{HAL Id: hal-00765061 \\ https://hal.inria.fr/hal-00765061}

Submitted on 12 Mar 2019

HAL is a multi-disciplinary open access archive for the deposit and dissemination of scientific research documents, whether they are published or not. The documents may come from teaching and research institutions in France or abroad, or from public or private research centers.
L'archive ouverte pluridisciplinaire HAL, est destinée au dépôt et à la diffusion de documents scientifiques de niveau recherche, publiés ou non, émanant des établissements d'enseignement et de recherche français ou étrangers, des laboratoires publics ou privés. 


\title{
Building a Global Time on Parallel Machines *
}

\author{
Jean-Marc JEZEQUEL \\ I.R.I.S.A. Campus de Beaulieu \\ F-35042 Rennes Cedex, FRANCE
}

\begin{abstract}
This paper presents a pragmatic algorithm to build a global time on any distributed system, which is optimal for homogeneous parallel machines. After some discution on time, clocks and distributed systems, we survey and criticize the classical approaches based on clock synchronisation techniques. Satisfying better our purposes, a statistical method is chosen as a building block to derive an original algorithm valid for any topology. This algorithm is particularly well suited for distributed algorithm experimentation purposes because, after an acquisition phasis, it induces neither CPU nor message overhead. We provide in the conclusion some data about its behavior and performances on some parallel machines.
\end{abstract}

\section{Introduction}

Whereas ordering two events occuring at the same place is straightforward, there is a problem if the events occur at different places, or if we want to compare various durations, because usually there is no common time reference among them.

In a distributed system, a common time reference (i.e. a global time) is very useful for two kinds of reasons. First, a global time availability allows to design simpler distributed algorithms to deal with synchronized behaviors, real-time constraints (like timeout for protocols) or actual ordering of events (Distributed Database Systems, version management...). Then, if we want to observe the behavior of a distributed algorithm on a distributed system (for test or debug or other purposes), a global time allows us to measure its performances, to observe the order of events, and to verify easily some properties (mutual exclusion...).

This paper discusses the way such a global time may be actually constructed on parallel machines. We define in section 2 some vocabulary and precise what kind of systems we are interested in. Section 3 is an overview of the software clock synchronization principles, methods and limits. To get rid of those limits (too constrainfull for our purposes), we present in section 4 a new hypothesis to justify an approach based on statistical estimations upon mutual dependencies of local clocks. Section 5 shows how this approach can be used as a building block to construct a global time in any distributed system, and that for some of them (the subclass of homogeneous parallel machines) this global time has the real time accuracy. We conclude with some practical results for this global time for some parallel machine, and on the interests and limits of this new service.

* This work has been partially supported by the French program on parallelism of the CNRS/C ${ }^{3}$, and within the ADP research team of the IRISA laboratory. 


\section{General Framework}

\subsection{About Time}

Agreeing with [10] we can define an event as a point on the time line, and a duration as the interval between two events. The real number $t(e)$ is associated to event $e$ by the fonction time. We call clock any abstract device which is able to measure durations. A physical clock is a device which can count the occurrence of quasi-periodic events. Such events are generally the observable oscillations of some physical system where the variations of a state variable of the system (position, volume, electrical tension...) are related to time through a periodic physical law. The granularity of a physical clock is the duration $g$ between two incrementations of the clock. The local time $l_{i}$ is the continuous time generated by a physical clock $C_{i}$, taking as time basis its average granularity $g_{i}$. We can say that $l t_{i}=g_{i}\left(C_{i}+e_{i}\right)$, where $e_{i}$ is the "reading error" of the discrete clock $C_{i}$.

\subsection{Distributed systems}

In the following we consider that a distributed system is a set of processors (or sites, machines, nodes) communicating only by messages transmission through a point to point communication network. We call parallel machine any homogeneous distributed system built on a local network. The transmission delays of messages are not negligible in front of internal action durations on a processor. Massively parallel machines like hypercubes or local networks of workstations are good examples of distributed systems. In order to compare different synchronization algorithms on those machines, let us present some typical data about some parallel machines: a network of Sun workstations (located on various buildings), linked with ethernet (and optical couplers between buildings); an Intel hypercube iPSC/2, with 64 processors (80386) linked by special hardware; and a FPS hypercube T-40, with 32 processors (Transputers). Measures have been performed with the ECHIDNA system, which provides an homogeneous interface for an high level programming language (Estelle) on parallel machines (see [9] for a presentation).

\begin{tabular}{|l|r|r|r|}
\hline Machines & Sun & iPSC/2 & FPS-T40 \\
\hline minimum transmission delay of a message $T_{\min }(\mathrm{ms})$ & 10 & 1 & 3 \\
\hline maximum transmission delay of messages $T_{\max }(\mathrm{ms})$ & $>100$ & $>10$ & $>20$ \\
\hline granularity of the available physical clock $g(\mathrm{~ms})$ & 20 & 1 & 0.064 \\
\hline physical clock medium drift $d(\mathrm{~s} /$ day) & 0 & $\approx 1$ & $\approx 0.5$ \\
\hline
\end{tabular}

\subsection{Physical clock for common processors}

Let us look into the usual way physical clock are built on common computers. There are two basic methods, the simpler (used in our Sun network) uses the 110 or $220 \mathrm{~V}$ power line to cause an interrupt on every voltage cycle (at 50 or $60 \mathrm{~Hz}$ ). The other one needs a local oscillator, which is commonly a (cheap) quartz crystal. The cut of the crystal determines its resonant frequency, within $50.10^{-6}$ of its nominal frequency for common commercial purposes $\left(1.10^{-6}\right.$ for military applications). This actual resonant frequency depends on the temperature and on few others factors of lesser importance. When such a crystal is mounted under tension, it generates a periodic signal which is fed into a counter to make it count down to zero. There, it causes a CPU interrupt (called clock tick): the CPU increments an internal register (its local "physical clock") and loads again the counter with the accurate predefined value. 
According to [5] and to various experiments made on our parallel machines, if the temperature is quite constant at each node then the trajectory of a local time generated by such a physical clock may be modeled with a constant frequency offset: as the frequency change rate is less than $10^{-7} /$ day, the resulting bias error on the time offset will be less than $30 \mathrm{~ns}$ for $10 \mathrm{~min}$. So, we can model the trajectory of a local time generated by such a physical clock with: $l t(t)=\alpha+\beta t+\delta(t)$, where $\alpha$ is the time offset at $t=0, \beta$ is the drift of the logical clock, $\beta=1+\kappa, \kappa=\frac{\Delta F}{F}$ (frequency offset) and $\delta(t)$ modelizes random perturbations and granularity.

\subsection{Global time and logical clock}

On each site of a distributed system there is such a physical clock showing a different time. Our goal is to build on each site a logical clock such that all logical clocks show the same hour at the same time.

We call global logical clock $(L C)$ an application from $\Re$ to $\Re^{n}$, such that:

$L C_{i}(t)$ is the value of the $i^{t h}$ component of $L C$ at time $t$

Increasing $\forall i \in[1 . . n], \forall t \in \Re, \forall d>0 \quad L C_{i}(t+d)-L C_{i}(t) \geq 0$

Agreement $\exists \epsilon \in \Re^{+}, \forall i, j \in[1 . . n], \forall t \in \Re \quad\left|L C_{i}(t)-L C_{j}(t)\right|<\epsilon$

We call global time the time $\mathrm{T}$ generated on each site by the component $L C_{i}$. The imprecision of the global time is $\epsilon$, and $G=2 \epsilon$ is its granularity.

As the major interest of a clock is to measure durations and to allow ordering of events, we are interested in the following properties for our logical clocks:

Accuracy $\exists \rho \in \Re, \forall t_{1}, t_{2} \in \Re \times \Re, \forall i \in[1 . . n]$

$\left(t_{2}-t_{1}\right)(1-\rho)<L C_{i}\left(t_{2}\right)-L C_{i}\left(t_{1}\right)<\left(t_{2}-t_{1}\right)(1+\rho)$

i.e. the logical clock is within a linear envelope of real time. If $L C_{i}$ is derivable, this property is equivalent to $\left|\frac{d L C_{i}(t)}{d t}-1\right|<\rho$.

\section{Internal causality}

$\forall e_{i}, e_{j}$ internal events of a distributed system $e_{i} \rightarrow e_{j} \Rightarrow L C_{i}\left(t\left(e_{i}\right)\right)<L C_{j}\left(t\left(e_{j}\right)\right)$

There exist known hardware solutions to build systems having such a global time, using phaselock loops or satellite synchronization. Besides the fact that such machines are no longer distributed systems, those solutions are quite expensive and not currently available for common parallel machines. So we have to check for software solutions, which have aroused a profuse bibliography.

\section{Software Clock Synchronization}

\subsection{Principles and Problems}

The usual way to synchronize two clocks $C_{i}$ and $C_{j}$ consists in choosing one of them (say $C_{i}$ ) as a reference (i.e. $\left.\forall t, L C_{i}(t)=C_{i}(t)\right)$, and then to measure the offset $\Delta_{i j}$ between $l t_{i}$ and $l t_{j}$ in order to set $L C_{j}(t)=C_{j}(t)-\Delta_{i j}$.

But, as clocks have a granularity, we can only measure $\Delta_{i j}=C_{j}-C_{i}$ in place of $l t_{j}-l t_{i}$, and as a frequency offset may exist between clocks, $l t_{i}$ and $l t_{j}$ can drift, thus $\Delta_{i j}$ is time dependent. Furthermore, in distributed systems the evaluation of $\Delta_{i j}$ is not trivial, because any information interchange can only be done through messages: site $P_{i}$ 
has to send to site $P_{j}$ the value at time $t$ of its clock, $C_{i}(\mathrm{t})$. As the transmission time $t m_{i j}$ of a message from $P_{i}$ to $P_{j}$ can't be known a priori, the error on the evaluation of $\Delta_{i j}$ can be as large as the maximum transmission time of a message.

It appears clearly that this method can be used only if transmission delays, granularities and frequency offsets are small enough with respect to the wanted agreement on the global time. Otherwise, we have to look for more sophisticated algorithms.

\subsection{Lamport's logical clocks}

Assuming the following hypothesis,

LH1 For every processor $P_{i}$ having available a physical clock $C_{i},\left|\frac{d C_{i}(t)}{d t}-1\right|<\kappa$

LH2 there exists a bound on the transmission delays of messages: $t_{d e l}=\mu+\xi$, where $\mu$ is the minimum delay of a message, and $\xi$ the known bound on the unpredictable delay of messages.

LH3 every $\tau$ seconds a message is sent over every edge of the network

LH4 physical clock granularity is thin enough to timestamp two events of the same site with different values

Lamport presents in [13] an algorithm to build synchronous logical clocks, which can be adapted to synchronize physical clocks: as long as $P_{i}$ doesn't receive any message, let $\frac{d L C_{i}(t)}{d t} \equiv \frac{d C_{i}(t)}{d t}$. But, upon sending a message, $P_{j}$ timestamps it with the value $T_{j}=L C_{j}(t)$; and when $P_{i}$ receives a message, it sets $L C_{i}(t)=\max \left(L C_{i}(t), T_{j}+\mu\right)$

The properties of those logical clocks are:

LP1 $\forall i, j \forall t\left|L C_{i}(t)-L C_{j}(t)\right|<\epsilon \approx 2 \kappa \tau+\xi$ which is for our examples: $\epsilon_{\text {Sun }}>50 m s, \epsilon_{i P S C}>$ $10 \mathrm{~ms}, \epsilon_{F P S}>20 \mathrm{~ms}$

LP2 $\left|\frac{d L C_{i}(t)}{d t}-1\right|<\kappa$ between resets, but undefined during resets.

But we can remark that the resulting global time doesn't stay within a linear envelope of real time, because clocks are always reset forward, each $\tau$ in the bad case. On the other hand, if $\tau$ is large then $\epsilon$ is large, and thus the agreement poor.

Anyway, if the application doesn't send enough messages to complete LH3, additional messages are needed, and thus the application can be perturbed. Furthermore, granularity is not really taken into account, meanwhile in our systems LH4 is not valid (excepted for the FPS).

\subsection{Improvements and limits}

Numerous researchers improve the Lamport's idea: among them, Lamport himself in [14] (where clocks are no longer always reset forward, and can tolerate some faults), and Marzullo who formalizes the problem and its solution in [16]. Various algorithms are presented to deal with byzantine behaviors in $[15,8]$ and in [19](optimal solution with respect to the Accuracy property: its global time accuracy is as good as the harware one (quartz)); see [17] and [18] for an overview and a detailed comparison.

But, as it is highlighted in $[11,12]$, those algorithms are quite complex, and thus difficult to implement correctly and maintain, and exhibit high CPU and messages overheads as the number of tolerated faults increases. Furthermore, it is observed in [2] that the overall increase in reliability provided by those byzantine algorithms is not always significant, compared to other sources of system failure. Thus, restricting the class of faults to be resisted, [2] presents a simpler algorithm, which improves the precision of 


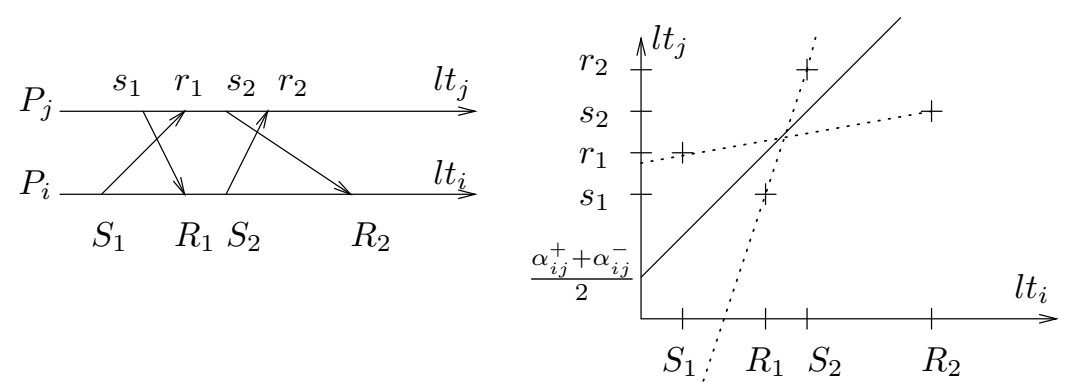

Figure 1: Statistical estimation of time and frequency offset

the global time (the trick is to lower $t_{d e l}=\mu+\xi$ (and thus $\epsilon$ ), and to consider that a message whose transfer delay is greater than $t_{d e l}$ is faulty).

So, apart for the accuracy and the fault tolerance problems, the primary solution of [13] has not been drastically improved. When extra messages are exchanged to control the mutual drift of physical clocks, the observational purpose of a global time is clearly unusable: those extra messages perturb the application that we want to observe. Furthermore, the precision of the global time is still not very good, because it depends closely on the variability of the transmission delays of messages, which is always the main factor of uncertainty. But, according to hypothesis LH1, LH2, LH3, [3] shows that much better results are impossible. So, if we want to go further, we have to study new hypothesis, reformulate the problem and present new methods.

\section{Statistical Estimation of Clock Offsets}

\subsection{Elimination of the transmission delay uncertainty}

In a first step, we are not interested in fault tolerance (in parallel machines, almost all faults are software bugs or fail stops). Furthermore, we think that clock synchronization is not an intrinsic problem of fault tolerance. As it is explicitely stated in [2], the major contribution brought by thoses techniques is to get rid of the transmission delay uncertainty (and eventually to deal with crash and join problems).

But transmission delays can be modelized by a random variable whose distribution is unknown, because they depend on the software overhead to access the network on the sending machine, on the network transmission time (depending on the size of the messages) and on the software overhead to deliver the incoming messages to the right tasks on the receiving machine, etc...

Reference [6] proposes a statistical method to eliminate this uncertainty when drift between clocks may be assumed negligible for short periods. Instead of performing only one message exchange when a resynchronization is needed, the algorithm performs numerous exchanges and selects the one with the best transmission delay to compute the time offset between two sites (and the precision of this evaluation).

The major advantage of this algorithm is its precision obtained on the global time, which is only limitated by the granularity of the physical clocks and the anisotropy of the network (i.e. difference between minimum transmission delays).

However, if the mutual drift between physical clocks is not negligible, frequent resyn- 
chronizations will be needed, and numerous messages added to the application: this global time is no longer suitable for observation purposes.

Until now we supposed that a bound was known on the mutual drift between physical clocks. According to this bound, a resynchronization round was triggered to compute new time offsets when the possible uncertainty due to mutual drifts became too high. The only way to avoid those resynchronization rounds would be to actually compute the frequency offset between physical clocks.

\subsection{Frequency Offset Evaluation}

The idea of [4] is also to use multiple message exchanges between $P_{i}$ and $P_{j}$ to estimate with a statistical method the time and the frequency offset between two sites. As for each site $P_{i}$ we have $l t_{i}(t)=\alpha_{i}+\beta_{i} t+\delta_{i}$ (see 2.3), there exist $\alpha_{i j}$ and $\beta_{i j}$ such that $l t_{j}(t)=\alpha_{i j}+\beta_{i j} l t_{i}(t)+\beta_{i j} \delta_{i j}$, where $\delta_{i j}$ is a random variable whose density function is the convolution between $\delta_{j}$ and $\delta_{i}$ density functions $\left(\beta_{i j}=\beta_{j} / \beta_{i} \quad \alpha_{i j}=\alpha_{j}-\beta_{i j} \alpha_{i}\right.$ and $\delta_{i j}=$ $\left.\delta_{j}-\beta_{i j} \delta_{i}\right)$. The actual purpose of [4] is thus to compute bounds for $\alpha_{i j}$ and $\beta_{i j}$.

Let $S_{i}^{k}$ be the event of site $P_{i}$ sending a message to $P_{j}, R_{j}^{k}$ the corresponding reception on $P_{j}$, and $\tau_{i j}$ the positive random variable (whose distribution is unknown) modelizing the transmission delay of messages. Thus, we can write for messages received by $P_{j}$ (see figure 1):

$$
l t_{j}\left(t\left(R_{j}^{k}\right)\right)=l t_{j}\left(t\left(S_{i}^{k}\right)+\tau_{i j}\right)=l t_{j}\left(t\left(S_{i}^{k}\right)\right)+\beta_{j} \tau_{i j}=\alpha_{i j}+\beta_{i j} l t_{i}\left(t\left(S_{i}^{k}\right)\right)+\beta_{j} \tau_{i j}+\delta_{i j}
$$

and symetricaly for messages sent by $P_{j}$ :

$$
l t_{j}\left(t\left(S_{j}^{k}\right)\right)=l t_{j}\left(t\left(R_{i}^{k}\right)-\tau_{j i}\right)=l t_{j}\left(t\left(R_{i}^{k}\right)\right)-\beta_{j} \tau_{j i}=\alpha_{i j}+\beta_{i j} l t_{i}\left(t\left(R_{i}^{k}\right)\right)-\beta_{j} \tau_{j i}+\delta_{i j}
$$

If $\delta_{i j}$ (mainly the granularity) is small in comparison with transmission delays $\left(\delta_{i j} \ll \tau_{i j}\right.$ so $\beta_{j} \tau_{i j}+\delta_{i j}>0$ and $\left.-\beta_{j} \tau_{j i}+\delta_{i j}<0\right)$, then the wanted line $\left(l t_{j}=\alpha_{i j}+\beta_{i j} l t_{i}\right)$ geometrically lies between the two separate sets of points $\mathrm{UP}=\left(S_{i}^{k}, R_{j}^{k}\right)$ and $\mathrm{LOW}=\left(R_{i}^{k}, S_{j}^{k}\right)$; see figure 1. Thus, we can compute higher and lower bounds $\alpha_{i j}^{+}, \alpha_{i j}^{-}$and $\beta_{i j}^{+}, \beta_{i j}^{-}$for $\alpha_{i j}$ and $\beta_{i j}$ using a geometrical algorithm ${ }^{1}$. Furthermore, as the duration and the number of points of the estimation increase, $\beta_{i j}^{+}-\beta_{i j}^{-}$decreases to 0 , and $\alpha_{i j}^{+}-\alpha_{i j}^{-}$to the difference between minimum transmission delays in the two directions. Practically, this convergence is very fast for distributed systems built on local networks, because the transmission delay distribution has an important mass near the minimum (see [7] for details and proof).

Hence, the problem of transmission delay variability is solved, and as the mutual frequency offset is computed instead of being bounded, resynchronization is no longer needed. This last property is very interesting because, after an acquisition phasis, this algorithm induces neither CPU nor message overhead. The application is thus no longer perturbed by the global time construction.

In the following, we will select this statistical method as a building block to derive an original algorithm valid for any topology.

\footnotetext{
${ }^{1}$ The first idea was to use a linear regression to estimate $\alpha_{i j}$ and $\beta_{i j}$, but [4] shows that this method can't give $100 \%$ confidence intervals.
} 


\subsection{Optimisations and limits for parallel machines}

First, let us see how we can optimize this method for parallel machines.

Let $\mu$ be a constant less than the minimum transmission delay (more precisely, $\mu$ is such that $\forall i, j \quad 0<\mu<\beta_{j} \tau_{i j}$ ), and $\delta$ such that $\forall i, j \quad\left|\delta_{i j}\right|<\delta . \delta \approx g$ (the granularity) because $\left|\delta_{i j}\right|=\left|\delta_{i} \otimes \delta_{j}\right|<2 \max \left(\left|\delta_{i}\right|\right)$, and $\left|\delta_{i}\right| \approx g / 2$ is mainly the precision of the physical clock $(\otimes$ is the convolution product).

A problem arises when the granularity is not small with respect to transmission delays $\left(\delta>\mu\right.$, see the Sun network example): the two sets of points $\left(S_{i}^{k}, R_{j}^{k}\right)$ and $\left(R_{i}^{k}, S_{j}^{k}\right)$ may overlap, because $\beta_{j} \tau_{i j}+\delta_{i j}$ is no longer always greater than $-\beta_{j} \tau_{j i}+\delta_{j i}$.

The solution to this problem consists in artificially increasing the minimum transmission delay so that $\mu^{\prime}>\delta$. Geometrically, this leads to move away the two sets of points eachother. The required minimum value of this adjustment is thus $\nu=\delta-\mu$, so that $\nu+\beta_{j} \tau_{i j}+\delta_{i j}>-\nu-\beta_{j} \tau_{j i}+\delta_{j i}$.

We can use the same method to decrease the value of the minimum transmission delay downto $\left|\mu_{i j}-\mu_{j i}\right|$ (i.e. the difference between minimum transmission delays in both directions) as long as the two sets of points $\left(S_{i}^{k}, R_{j}^{k}\right)$ and $\left(R_{i}^{k}, S_{j}^{k}\right)$ remain separated each other, i.e. the adjustment $\nu$ is such that $\nu>\delta-\mu$ (if $\nu>0$ the sets are moved away and else they are bring together).

This can be used to gain precision on the evaluation of $\beta_{i j}$ and $\alpha_{i j}$ with the same acquisition period $\Delta T$, as precision on $\alpha_{i j}$ depends on the precision on $\beta_{i j}$ (and is limited by $\left.\left|\mu_{i j}-\mu_{j i}\right|\right)$, and the lower bound for $\Delta \beta_{i j}=\frac{1}{2}\left(\beta_{i j}^{+}-\beta_{i j}^{-}\right)$is $\frac{2 \mu}{\Delta T}$.

If the network is highly symmetrical and homogeneous (as it is in our three examples), minimum transmission delays in both directions can be considered equal (isotropic networks), and thus lower bound for $\Delta \beta_{i j}$ is $\frac{2(\mu+\nu)}{\Delta T}=\frac{2 \delta}{\Delta T}$.

\section{$5 \quad$ Building a global time over any connected network}

\subsection{Theoretical solution}

At this point, a site is able to evaluate its local linear dependencies with its neighbours. From those local dependencies, we want to derive some global dependencies in order to build on each processor a global time function $T G_{i}\left(l t_{i}(t)\right)$ having the required properties.

We assume in this part that each processor $P_{i}$ of the network $N$ has computed (in parallel) its linear dependencies with all of its neighbours with the algorithm described in the previous part (let us call it A1). So, the following system (S) of inequations holds:

$$
\forall P_{i} \in N, \forall P_{j} \in V_{i}, \alpha_{i j}^{-}<\alpha_{i j}<\alpha_{i j}^{+}, \beta_{i j}^{-}<\beta_{i j}<\beta_{i j}^{+} \text {and } l t_{j}(t)=\alpha_{i j}+\beta_{i j} l t_{i}(t)+\delta_{i j}
$$

where $V_{i}$ denotes the set of $P_{i}$ 's neighbours. As $\left|\delta_{i j}\right| \approx g$, we have:

$$
\forall P_{i} \in N, \forall P_{j} \in V_{i}, \alpha_{i j}^{-}-g+\beta_{i j}^{-} l t_{i}(t)<l t_{j}(t)<\alpha_{i j}^{+}+g+\beta_{i j}^{+} l t_{i}(t)
$$

As $l t_{i}(t)=\alpha_{i}+\beta_{i} t+\delta_{i}$, and $\left|\delta_{i}\right| \approx g / 2$, (S) is equivalent to

$\forall P_{i} \in N, \forall P_{j} \in V_{i}, \alpha_{i j}^{-}-g+\beta_{i j}^{-}\left(\alpha_{i}-g / 2\right)+\beta_{i j}^{-} \beta_{i} t<\alpha_{j}+\beta_{j} t<\alpha_{i j}^{+}+g+\beta_{i j}^{+}\left(\alpha_{i}+g / 2\right)+\beta_{i j}^{+} \beta_{i} t$

This is valid for each $t$, so:

$\forall P_{i} \in N, \forall P_{j} \in V_{i}, \beta_{i j}^{-} \beta_{i}<\beta_{j}<\beta_{i j}^{+} \beta_{i} ; \alpha_{i j}^{-}-g+\beta_{i j}^{-}\left(\alpha_{i}-g / 2\right)<\alpha_{j}<\alpha_{i j}^{+}+g+\beta_{i j}^{+}\left(\alpha_{i}+g / 2\right)$ 
As the method used in A1 is highly symetric, $\beta_{i j}^{+}=1 / \beta_{j i}^{-}$and $\alpha_{i j}^{+}=-\alpha_{j i}^{-} / \beta_{i j}^{-}$, so half of the inequations are redondant. We want to solve this system, i.e. to find intervals $\left[\alpha_{i}^{-}, \alpha_{i}^{+}\right]$and $\left[\beta_{i}^{-}, \beta_{i}^{+}\right]$for all $P_{i}$ that verify the system. There exists at least a solution: if every site has a linear dependency with all of its neighbours and if the graph is connected, every site has a linear dependency with every other by composition of the dependencies along any path. There exists even an infinity of solutions, each one being homothetic of the others (in terms of $\beta$ ).

The principle of the solution is to choose a reference site $P_{r}$ (either statically or by regular dynamical election), where we state $\beta_{r}^{-}=\beta_{r}^{+}=1$ and $\alpha_{r}^{-}=\alpha_{r}^{+}=0$; and then to eliminate all the inequations of $(\mathrm{S})$ by substitution.

Let $d(i, j)$ be the topological distance from $P_{i}$ to $P_{j}$ on $N$. Let $D_{r}(p)=\{j \in N \mid$ $d(r, j)=p$ be the set of sites which are at distance $p$ from $P_{r}$. We suppose that there exists a minimal spawning tree $T$ over $N$ (i.e. where distance from $P_{r}$ to $P_{j}$ along $T$ is $d(r, j))$, whose root is $P_{r}$ and diameter $d$, and that every process $P_{i}$ knows its depth and its neighbour's one on this tree ${ }^{2}$.

The system will be solved from near to near along $T$, i.e. for the neighbours of $P_{r}$, then for the neighbours of the neighbours etc...

We say that a site $P_{j}$ is synchronized if and only if all the inequations of (S) with terms in $\alpha_{i j}$ or $\beta_{i j}$ such that $d(r, i) \leq d(r, j)$ have been eliminated (so we have found the wanted intervals $\left[\alpha_{j}^{-}, \alpha_{j}^{+}\right]$and $\left.\left[\beta_{j}^{-}, \beta_{j}^{+}\right]\right)$. Let be $S^{p}$ the system where $\forall P_{j} \in \bigcup_{k \in[0, p]} D_{r}(k), P_{j}$ is synchronized.

Theorem If $P_{r}$ is the root of a minimal spawning tree $T$ and if $P_{r}$ is synchronized, then it is possible to eliminate all the inequations of $(S)$ in order to synchronize all the sites of $T$ in $d$ steps, where $d$ is the depth of $T$.

Demonstration (by induction on the depth of the graph):

Initially, only site $P_{r}$ is synchronized, and $S^{0}=(\mathrm{S})$. Suppose that: $\forall P_{j} \in$ $\bigcup_{k \in[0, p-1]} D_{r}(k), P_{j}$ is synchronized. The graph is synchronized until depth $p-1$, and remaining inequations form the system $S^{p-1}$. Let us see how to synchronize it at depth $p$.

Let be $P_{j} \in D_{r}(p)$ and $P_{i} \in V_{j}$. As $d(i, j)=1, V_{j} \subset D_{r}(p-1) \cup D_{r}(p) \cup D_{r}(p+1)$. So we have to eliminate all the inequations of $S^{p-1}$ having terms in $\alpha_{i j}$ or $\beta_{i j}$ such that $P_{i} \in D_{r}(p-1) \cup D_{r}(p)$.

1. If $P_{i} \in D_{r}(p-1)$, then $P_{i}$ is synchronized (by hypothesis), so there exist solutions such that $\alpha_{i}^{-}<\alpha_{i}<\alpha_{i}^{+}$and $\beta_{i}^{-}<\beta_{i}<\beta_{i}^{+}$. In $S^{p-1}$ we can extract the two following inequations (the symetric one, in terms of $\alpha_{j i}$ and $\beta_{j i}$ being equivalent to those one):

$$
\beta_{i j}^{-} \beta_{i}<\beta_{j}<\beta_{i j}^{+} \beta_{i} \text { and } \alpha_{i j}^{-}-g+\beta_{i j}^{-}\left(\alpha_{i}-g / 2\right)<\alpha_{j}<\alpha_{i j}^{+}+g+\beta_{i j}^{+}\left(\alpha_{i}+g / 2\right)
$$

This is valid for all $P_{i} \in V_{j} \cap D_{r}(p-1)$, so the conjonction of those inequations gives:

$$
\left[\beta_{j}^{-}, \beta_{j}^{+}\right]=\bigcap_{P_{i} \in V_{j} \cap D_{r}(p-1)}\left[\beta_{i}^{-} \beta_{i j}^{-}, \beta_{i}^{+} \beta_{i j}^{+}\right]
$$

\footnotetext{
${ }^{2}$ If every site can know statically the topology of $R$, then $T$ can be statically defined. Otherwise $T$ has to be built, using for example the algorithm (say A2) provided in [1], after whom each site broadcasts its depth in $T$ to its neighbours.
} 
and

$$
\left[\alpha_{j}^{-}, \alpha_{j}^{+}\right]=\bigcap_{P_{i} \in V_{j} \cap D_{r}(p-1)}\left[\alpha_{i}^{-}-g+\beta_{i j}^{-}\left(\alpha_{i}^{-}-g / 2\right), \alpha_{i}^{+}+g+\beta_{i j}^{+}\left(\alpha_{i}^{+}+g / 2\right)\right]
$$

2. If $P_{i} \in D_{r}(p)$, let us notice that $D_{r}(p)$ forms a sub-network $N_{p}$, partitioned in a set of connected sub-networks $N_{p}^{1} . . N_{p}^{n}$.

- If $N_{p}^{j}=\left\{P_{j}\right\}$, i.e. if $V_{j} \cap D_{r}(p)=\emptyset$, then there is no more inequations over $P_{j}$, so $P_{j}$ is synchronized.

- Otherwise, for each of those connected sub-networks $N_{p}^{k}$ we choose again (either statically or dynamicaly) a new reference site, root of a new minimal covering tree over $N_{p}^{k}$, and we synchronize those sub-networks with the same method as for the main network.

As the number of sites in $N_{p}^{k}$ is strictly less than the number of sites in $N$ (the reference site of $N$ can't be in $N_{p}^{k}$ ), this leads to build decreasing sequences (with the inclusion meaning) of unsynchronized sub-networks whose minimal size is one site. Upon synchronization of thoses last sites (a single site network is synchronized by definition), $N_{p}^{k}$ becomes synchronized, thus $P_{j} \in N_{p}^{j}$ is synchronized.

\subsection{Algorithm derivation}

From the mathematical resolution of (S), we can derive directly a distributed algorithm A3 to make the problem be solved by the considered distributed system.

$A 3\left(N, P_{r}\right):=--$ Algorithm to synchronize network $N$, with $P_{r}$ as reference ${ }^{3}$.

for each process $P_{j}$ do begin

$p:=\operatorname{distance}\left(P_{j}, P_{r}\right)$;

-- STEP 1, get the values from the already synchronized neighbours

if $p>0$ then begin

$$
\begin{aligned}
& \forall P_{i} \in V_{j} \cap D_{r}(p-1) \quad P_{i} ?\left(\left[\alpha_{i}^{-}, \alpha_{i}^{+}\right],\left[\beta_{i}^{-}, \beta_{i}^{+}\right]\right) ; \\
& {\left[\beta_{j}^{-}, \beta_{j}^{+}\right]:=\bigcap_{P_{i} \in V_{j} \cap D_{r}(p-1)}\left[\beta_{i}^{-} \beta_{i j}^{-}, \beta_{i}^{+} \beta_{i j}^{+}\right] ;} \\
& {\left[\alpha_{j}^{-}, \alpha_{j}^{+}\right]:=\bigcap_{P_{i} \in V_{j} \cap D_{r}(p-1)}\left[\alpha_{i}^{-}+\beta_{i j}^{-} \alpha_{i j}^{-}, \alpha_{i}^{+}+\beta_{i j}^{+} \alpha_{i j}^{+}\right] ;}
\end{aligned}
$$

end;

- STEP 2

if $V_{j} \cap D_{r}(p) \neq \emptyset$ then begin

Perform A2 $\left(N_{p}^{j}, P_{s}\right) ; \quad--$ to build a minimal covering tree (whose root is $P_{s}$ )

-- over the subnetwork $N_{p}^{k}$ (possible static knowledge)

Perform $A 3\left(N_{p}^{j}, P_{s}\right) ; \quad--$ recursive call end;

-- STEP 3, as $P_{j}$ is synchronized, it broadcasts its values deeper on the tree $\forall P_{i} \in V_{j} \cap D_{r}(p+1) \quad P_{i} !\left(\left[\alpha_{j}^{-}, \alpha_{j}^{+}\right],\left[\beta_{j}^{-}, \beta_{j}^{+}\right]\right) ;$ end;

\footnotetext{
${ }^{3}$ Hereafter, " $P_{i}$ ?" denotes the asynchronous reception of a message from $P_{i}$, and " $P_{i}$ !" the emission to $P_{i}$.
} 
So, the full algorithm is:

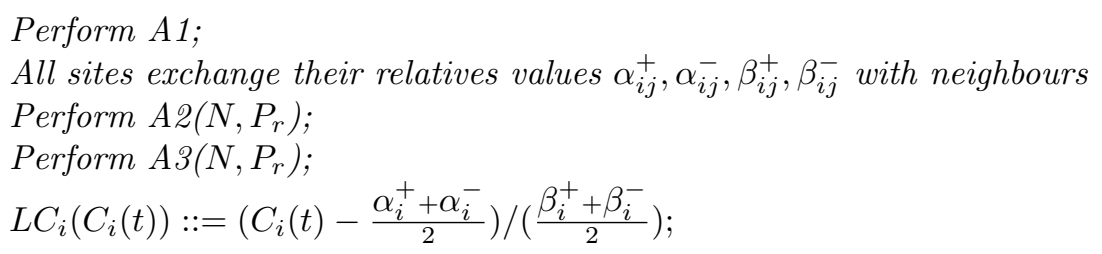

\subsection{Application to some classical topologies}

Our algorithm doesn't need to know statically the actual topology of the network. But if it is known, we can derive from it simpler algorithms to suit the particularities of the network, because the second step of the general algorithm (which could be quite costly) will be streched.

Fully connected network In such a network, the depth of the covering tree is always 1. So, all sites are chosen one after the other to be the next reference site for the remaining subnetwork. During the STEP 1 of A3, the only message expected is from the reference site of the current subnetwork, and the STEP 3 is performed only by the site which is reference of its subnetwork. Supposing that A2 is not actually performed (static order is known to choose next reference site), the time complexity of A3 is the complexity on the last site chosen: $O(n)$. We can notice that we get there a distributed version of the algorithm first presented in [4].

Ring topology Let be $R_{n}$ a network of $\mathrm{n}$ processors connected with a ring topology. We must study two cases, depending on the parity of $n$. If $n$ is even, $\forall P_{j} \in$ $R_{n}, \quad V_{j} \cap D_{r}(p)=\emptyset$. So, the second step of A3 becomes useless, and its time complexity is $O(n / 2)$.

But if $n$ is odd, the two sites located at distance $\frac{n-1}{2}$ of $P_{r}$ are neighbours. So, the second step of A3 is usefull only for those two sites, and time complexity is also $O\left(\frac{n}{2}\right)$.

Star topology If we choose the center of the network as the reference site, the minimal covering tree depth is only one, and thus A3 complexity becomes $O(1)$.

Hypercube topology Let be $P_{r}$ a site of an hypercube H, whose dimension (and thus diameter) is $d$. Hypercube topologies have numerous interesting particularities. Among them, if $P_{s} \in D_{r}(p)$, then $V_{s} \cap D_{r}(p)=\emptyset$ : a site which is at distance $p$ from $P_{r}$ has no neighbour at the same distance $p$ from $P_{r}$.

So, in the same way that for an even ring, the second step of A3 becomes useless, and time complexity is $O(d)$.

\subsection{Global time and real time}

As we have chosen the physical clock on one site to synchronize all the others, the resulting global time can't be better (in terms of accuracy) than the local time generated by the quartz of the reference site. This is generally not a problem when we are only interested in internal events observation. However, if we have to deal with external events 
references, a synchronization with a better external clock might be required. If such a clock is connected to the network, it is possible to select its site as the primary reference for our algorithm, so the accuracy of the global time is its accuracy.

However in almost all parallel machines, such a good clock is not available. But on homogeneous networks (such as hypercube machines), the available quartz have a resonant frequency that can be modelized on the set of all the quartz of the network by a random variable whose mean value is the nominal frequency of the quartz. So, $\frac{1}{N} \sum_{i=1}^{N} \beta_{i}=1 \pm \varepsilon, \quad \varepsilon \approx 10^{-10}$.

Thus it is possible to append to our algorithm a last phase where it adjusts all the computed frequency offset with their mean value. We can then have an accuracy very close to real time, without any reference to a better than quartz external clock (e.g. atomic).

\section{Conclusion}

The algorithm described above has been simplified for the hypercube topology and specified with the Estelle programming language in order to be compiled with ECHIDNA and experimented on Sun network, on iPSC/2 and on FPS-T40.

An initial acquisition period of $300 \mathrm{~s}$ yields to the following results:

\begin{tabular}{|r|r|r|r|}
\hline Machines & Sun & iPSC $/ 2$ & FPS-T40 \\
\hline Physical clock granularity $g(\mathrm{~ms})$ & 20 & 1 & 0.064 \\
\hline Measured bound on $\Delta F / F$ & 0 & $2.10^{-5}$ & $5.10^{-6}$ \\
\hline Best precision with classical methods $(\mathrm{ms})$ & 80 & 10 & 20 \\
\hline Precision on $\beta_{i}$ & $6.10^{-5}$ & $4.10^{-6}$ & $5.10^{-7}$ \\
\hline Initial precision of $L C_{i}(\mathrm{t})(\mathrm{ms})$ & 50 & 2 & 0.2 \\
\hline Precision after one hour $(\mathrm{ms})$ & 200 & 15 & 2 \\
\hline
\end{tabular}

The hypothesis of linearity between physical clocks (i.e. that frequency offset rate is actually negligible) has been verified for rather large periods $(\approx 12 h)$ through various experiments. However, the imprecision of our global time increases linearly with time: $G \approx 2 g+2 \Delta \beta t \approx 2 g+\frac{4 \delta}{\Delta T} t$. So, if this algorithm is to be used continuously, resynchronization rounds should take place dynamically when the precision becomes too bad for the current purpose, or application messages can be used to enforce the precision on frequency mutual offsets during the application execution.

The granularity of our global time doesn't always allow us to check for internal causality, i.e. that messages are always sent before they are received (at least for the Sun and the iPSC). But, as shown by the FPS example, this is mostly an hardware problem, and there is no software possibility to get rid of physical clock granularity. However various experiments showed that our global time can be used to order (with high probability) communication events, even on the iPSC. But, if we would use this new service in the property verification field, it would be interesting to study the idea of a probabilistic order of events, in place of classical partial orders.

For the distributed algorithm experimentation purpose, this global time algorithm has been integrated in the ECHIDNA system (along with the Lamport's algorithm to ensure internal causality), so it is now possible to observe a distributed algorithm behavior on a parallel machine without perturbing it. 


\section{Acknowledgements.}

Special acknowledgment is due to Y. Haddad for the original idea of frequency offset estimation, to C. Jard for his constant support and advices, and to all the members of the ADP team for their reviewing.

\section{References}

[1] A. T. Cheung. Graph traversal techniques and the maximum flow problem in distributed computing. IEEE Trans. on SE, SE-9(4):504-512, 1983.

[2] F. Cristian, H. Aghili, and R. Strong. Clock synchronization in the presence of omission and performance faults, and processors joins. In Proc. of 16th IEEE Symposium on FaultTolerant Computing Systems, Vienna, pages 218-223, July 1986.

[3] D. Dolev, J.Y. Halpern, and R. Strong. On the possibility and impossibility of achieving clock synchronization. In Proc. of 16th ACM Symposium on Theory of Computing, pages 504-511, April 1984.

[4] A. Duda, G. Harrus, Y. Haddad, and G. Bernard. Estimating global time in distributed system. In Proc. 7th Int. Conf. on Distributed Computing Systems, Berlin, 1987.

[5] C.E Ellingson and R.J. Kulpinski. Dissemination of system time. IEEE Transactions on Communications, COM-21(5):605-623, May 1973.

[6] R. Gusella and S. Zatti. A network time controler for a distributed berkeley UNIX system. IEEE Distr. Proc. Tech. Comm. Newsletter, SI-2(6):7-15, June 1984.

[7] Y. Haddad. Performance dans les systèmes répartis: des outils pour les mesures. Thèse de Doctorat, Univ. Paris-Sud, Centre Orsay, PARIS, Septembre 1988.

[8] J.Y. Halpern, B. Simons, R. Strong, and D. Dolev. Fault-tolerant clock synchronization. In Proc. of the Third ACM Symposium on Principles of Distributed Computing, Vancouver, Canada, pages 89-102, August 1984.

[9] C. Jard and J.-M. Jézéquel. A multi-processor Estelle to $C$ compiler to experiment distributed algorithms on parallel machines. In Proc. of the $9^{\text {th }}$ IFIP International Workshop on Protocol Specification, Testing, and Verification, University of Twente, The Netherlands, North Holland, 1989.

[10] H. Kopetz. Accuracy of time measurement in distributed real time systems. In 5th Symposium on Reliability in Distributed Software and Database Systems, pages 35-41, IEEE Comp. Society, 1986.

[11] H. Kopetz and W. Ochsenreiter. Clock synchronization in distributed real time systems. In IEEE Transaction on Computers, Special issue on Real Time Systems, pages 933-940, August 1987.

[12] C.M. Krishna and K.G. Shin. Synchronization and fault-masking in redundant real time systems. In Proc. of the FTCS 14, IEEE Press, pages 151-157, 1984.

[13] L. Lamport. Time, clocks and the ordering of events in a distributed system. Communications. of the ACM, 21(7):558-565, July 1978.

[14] L. Lamport and P.M. Melliar-Smith. Synchronizing clocks in the presence of faults. Journal of the ACM, 32(1):52-78, Juanary 1985.

[15] J. Lundelius and N. Lynch. A new fault-tolerant algorithm for clock synchronization. In Proc. of the Third ACM Symposium on Principles of Distributed Computing, Vancouver, Canada, pages 75-88, August 1984

[16] K. Marzullo and S. Owiki. Maintaining time in a distributed system. In ACM Operating Systems Rev., pages 44-54, 1983.

[17] F.B. Schneider. A paradigm for reliable clock synchronization. In Proc. Advanced Seminar Real Time Local Area Network, pages 85-104, April 1986.

[18] B. Simons, J. Lundelius, and N. Lynch. An Overview of Clock Synchronization. Technical Report, IBM Research Division, October 1988.

[19] T.K. Srikanth and S. Toueg. Optimal clock synchronization. In 4th Annual ACM Symposium on Principles of Distributed Computing, pages 71-86, August 1985. 